\title{
Boundary element analysis of deformation and movement of a capsule and a red blood cell close to the wall
}

\author{
S. Nix ${ }^{1}$, Y. Imai ${ }^{2}$, T. Ishikawa ${ }^{2} \&$ T. Yamaguchi ${ }^{1}$ \\ ${ }^{1}$ Department of Biomedical Engineering, Tohoku University, Japan \\ ${ }^{2}$ Department of Bioengineering and Robotics, Tohoku University, Japan
}

\begin{abstract}
A capsule is defined as a liquid drop enclosed by an elastic membrane. The study of capsule behavior near a stationary surface has a number of biological applications, such as red blood cells in the cardiovascular system. However, near-wall behavior of capsules has not been established well. In this study, we investigate the motion of initially spherical and biconcave capsules in simple shear flow near an infinite plane using a boundary integral method coupled to a finite element method. We find that the deformation of a capsule depends on its initial shape, orientation, and capillary number $(\mathrm{Ca})$. However, the lift velocity of a capsule is dependent on its steady state deformation and distance from the wall. The dependence of lift velocity on deformation may help to explain phenomena such as leukocyte margination.

Keywords: boundary integral method, finite element method, capsule flow, lateral migration.
\end{abstract}

\section{Introduction}

Red blood cells (RBCs) have been observed to exhibit higher deformability than other cells in the blood, such as white blood cells (WBCs) [1]. Furthermore, while the concentration of RBCs in microcirculation is highest near the center of the vessel, WBCs are found disproportionately near the vessel wall, particularly at low shear rates [2]. Several mechanisms have been proposed for this difference in lateral migration, such as an exclusion effect by the RBCs on a WBC, as well as hydrodynamic forces. 
Previous theoretical studies on lateral migration near a plane wall were performed by Chaffey et al. [3] and Chan and Leal [4]. They found that, for a liquid drop, the rate of lateral migration depends on the drop deformation, the distance from the wall, and the viscosity ratio. The influence of these factors have been investigated numerically for a liquid drop [5,6], but little work has been done thus far on other types of particles.

Here, we focus on the effect of a plane wall on the migration of a capsule, or a liquid drop bound by a hyperelastic membrane. A capsule is a better model for a cell, particularly an RBC, due to the presence of a spectrin network that provides an elastic character to the RBC membrane. In addition, the characteristics of the phospholipid bilayer membrane, orders of magnitude thinner than the radius of the cell and resistant to area expansion, allow the membrane to be modeled as a two-dimensional material that is nearly incompressible. We focus on two types of capsules: initially spherical and initially biconcave. In addition, we consider the effect of varying flow conditions, quantified using the capillary number $(C a)$, and the initial orientation of the biconcave capsule.

Since only the capsule membrane and the wall are of interest, a boundary integral method (BIM) is employed, using the Green's function for a semiinfinite flow developed by Blake [7]. In addition, to track the deformation of the membrane, the surface is discretized and solved at each step using a constitutive law and the finite element method (FEM).

\section{Method}

The numerical method, a coupling of the boundary integral method and finite element method, was developed by Walter et al. [8] to simulate the motion of capsules in an infinite shear flow.

In this method, a constitutive law for a two-dimensional hyperelastic material is used to calculate the tension in the capsule membrane. The equilibrium between membrane tension and viscous traction of the inner and outer fluids is given by a variational approach. From the viscous traction, the velocity of the membrane is computed using the boundary integral equation.

\subsection{Algorithm}

To consider the motion of a red blood cell and an equivalent spherical capsule, the Skalak constitutive law [9] is employed. The strain energy function for the Skalak law is given by

$$
w_{s}=\frac{G_{s}}{2}\left(I_{1}^{2}+2 I_{1}-2 I_{2}+C I_{2}^{2}\right),
$$

where $G_{s}$ is the surface shear modulus and $C$ represents the resistance to area dilation. For a spherical capsule, the value $C=1$ is used, but since the red blood cell membrane is nearly incompressible, the area dilation modulus is increased to $C=10$. 
The membrane tension is written in terms of the strain energy by the relation

$$
\mathbf{T}=\frac{1}{J_{s}} \mathbf{F}_{\mathbf{s}} \cdot \frac{\partial w_{s}}{\partial \mathbf{e}} \cdot \mathbf{F}_{\mathbf{s}}{ }^{T},
$$

where $J_{s}$ is the area dilation ratio, $\mathbf{F}_{s}$ is the surface deformation gradient tensor, and $\mathbf{e}$ is the Green-Lagrange strain tensor. From the membrane tension, the equilibrium on the surface of the capsule is determined by the equation

$$
\int_{S} \hat{\mathbf{u}} \cdot \mathbf{q} \mathrm{d} S=\int_{S} \hat{\boldsymbol{\varepsilon}}_{S}: \mathbf{T} \mathrm{d} S,
$$

where $\hat{\mathbf{u}}$ is a virtual displacement that gives a virtual strain $\hat{\varepsilon}_{s}$, and $\mathbf{q}$ is the viscous traction on the membrane. This equation is solved by discretization into a linear system.

The velocity of the capsule surface in terms of the viscous load is given by the boundary integral equation,

$$
\mathbf{v}\left(\mathbf{x}_{0}\right)=\mathbf{v}^{\infty}\left(\mathbf{x}_{\mathbf{0}}\right)-\frac{1}{8 \pi \mu} \int_{S} \mathbf{J}\left(\mathbf{x}_{\mathbf{0}}, \mathbf{x}\right) \cdot \mathbf{q}(\mathbf{x}) \mathrm{d} S,
$$

where $\mathbf{v}$ is the velocity at a nodal point $\mathbf{x}_{0}, \mathbf{v}^{\infty}$ is the undisturbed flow, $\mu$ is the viscosity of the fluid, and $\mathbf{J}$ is the Green's function for a semi-infinite fluid given by Blake [7]. Here, the undisturbed flow $\mathbf{v}^{\infty}$ is a simple shear flow with a shear rate $\dot{\gamma}$.

\subsection{Numerical conditions}

A schematic of the initial conditions for a spherical capsule, showing the coordinate system as well as the key parameters, is shown in figure 1 .

The lengths in this computation, such as the initial distance from the wall $h$, are scaled by the radius $a$. For an initially spherical capsule, $a$ is equal to the

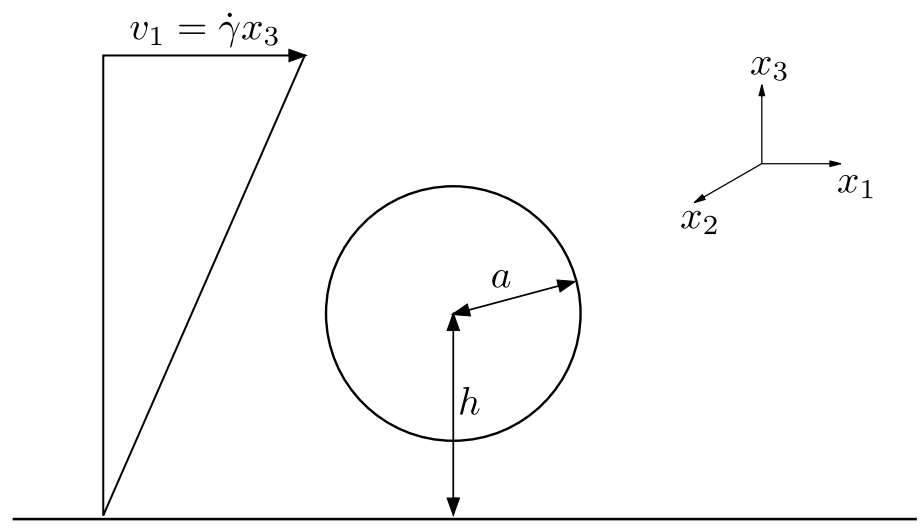

Figure 1: Schematic of computation. 

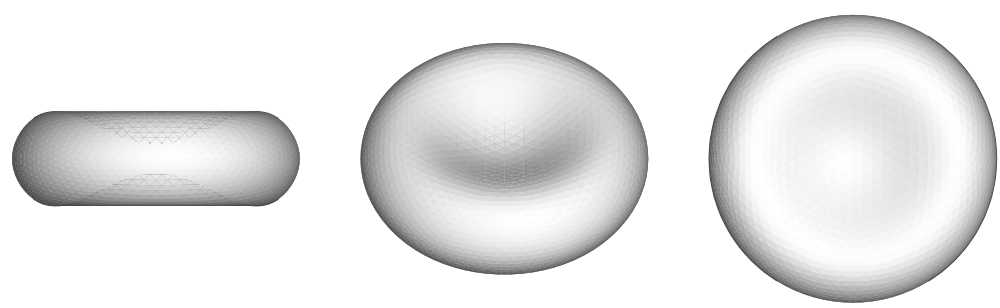

Figure 2: Initial orientations of biconcave capsules. Shear direction points to the right.

initial radius, whereas for a non-spherical capsule, $a$ is the radius of a spherical capsule with the same volume as the non-spherical capsule. A parameterization of the resting shape of an RBC is given by Evans and Fung [10], with the major axis $L_{1}$ in the initial state set to $1.386 a$ [11].

Since inertial effects are neglected, the flow condition is given by the capillary number,

$$
C a=\frac{\mu \dot{\gamma} a}{G_{s}}
$$

which shows the relative strengths of the viscous and elastic forces.

In addition, the initial orientation of the $\mathrm{RBC}$ is rotated in the direction such that the axis of rotation is parallel to the wall, as shown in figure 2 . The position on the left hand side of figure 2 is given by the angle $\theta_{0}=0$, and the RBC is rotated $\pi / 4$ and $\pi / 2$ to give middle and right positions, respectively.

\section{Results}

The length of the major axis in the shear plane normalized by the radius, $L_{1} / a$, is used to quantify the deformation of the capsules. In addition, the deformation, as well as the lift velocity, are compared using the average major axis length in an infinite flow, $L_{1}^{\infty} / a$.

\subsection{Deformation}

In figure 3, the deformation of spherical capsules is shown as the capsules move away from the wall. Two regimes are seen: first, a transient motion occurs as the capsule is stretched by the discontinuous start of the background flow. After the transient motion fades, the capsule reaches a quasi-steady state, as the deformation decreases at smaller rate than at the transient state. Interestingly, as the capillary number increases, the relative deformation of the capsule in the transient state also increases.

In figure 4, the deformation of RBCs with varying initial orientation and at $C a=1.15$ are shown as they move away from the wall. Unlike the spherical capsules, the RBCs show two distinct deformation profiles that depend on initial 


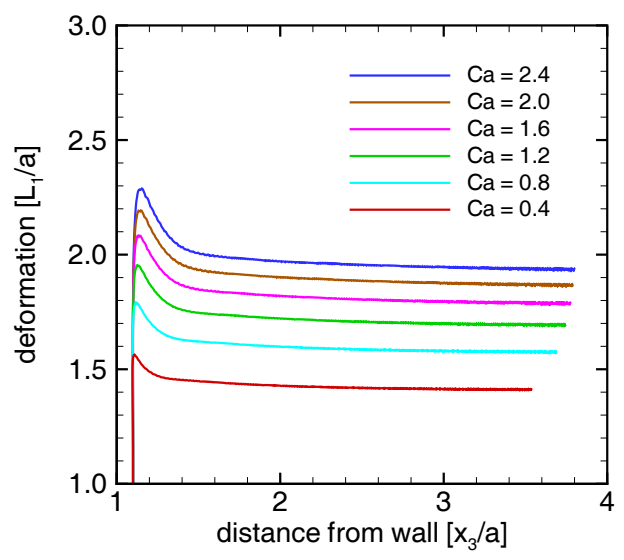

Figure 3: Deformation of spherical capsules.

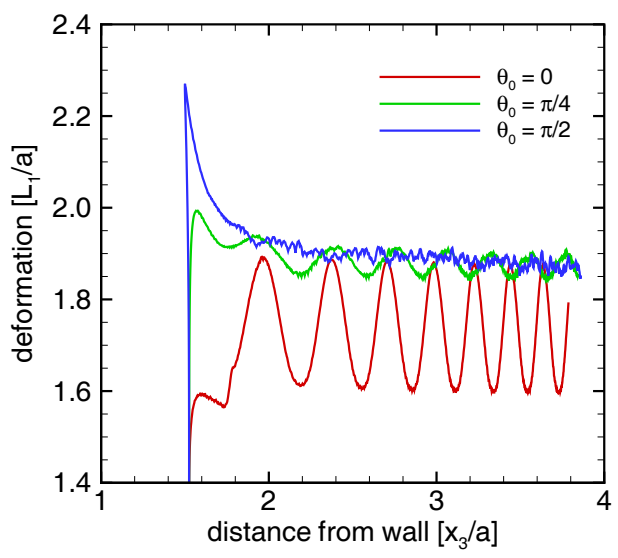

Figure 4: Deformation profile of RBCs.

orientation. When the RBC is initially symmetrical with respect to the shear plane $\left(\theta_{0}=\pi / 2\right)$, it exhibits position-dependent deformation similar to the spherical capsule. On the other hand, when the RBC is symmetrical in the plane parallel to the wall $\left(\theta_{0}=0\right)$, it experiences regular oscillations in its deformation profile, with a lower averaged value than the $\theta_{0}=\pi / 2$ case. The RBC with an initial orientation of $\theta_{0}=\pi / 4$ shows a combination of these two patterns; it exhibits regular oscillations in deformation, but with an average magnitude close to that of the $\theta_{0}=\pi / 2$ case.

In figure 5 , the peak relative deformations of the RBCs shown in figure 4 and a capsule at $C a=1.2$ are plotted together. The capsule and the RBC with an initial orientation of $\theta_{0}=0$ show a linear dependence between the peak and steady state 


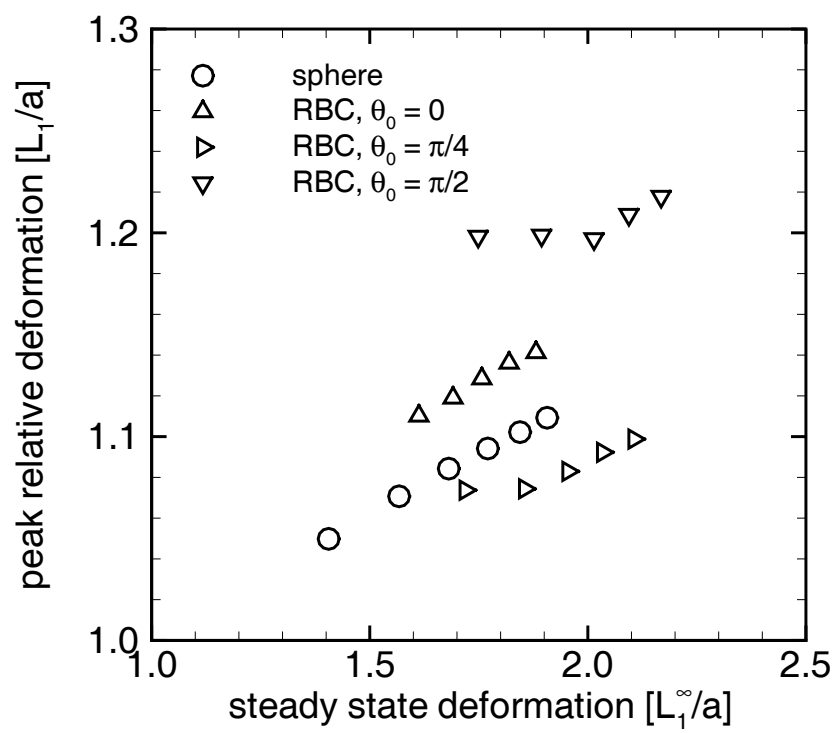

Figure 5: Peak deformation of a spherical capsule and RBCs.

deformations. On the other hand, the RBCs at $\theta_{0}=\pi / 4$ and $\theta_{0}=\pi / 2$ only show a linear dependence on the steady state deformation at a high peak deformation. At lower deformations, the peak deformation of these RBCs is nearly constant with respect to the steady state deformation.

\subsection{Lift velocity}

The lift velocity is defined as the velocity at which the capsule separates from the wall. Previous studies $[3,4]$ have predicted that a liquid drop exhibits a lift velocity proportional to $\left(a / x_{3}\right)^{2}$ at distances far from the wall.

To confirm whether the lift velocity of an initially spherical capsule shows the same dependence on $\left(a / x_{3}\right)^{2}$, the lift velocities for initially spherical capsules with varying initial positions are plotted on a log-log scale in figure 6. At distances far from the wall, the lift velocity of an initially spherical capsule approaches the $\left(a / x_{3}\right)^{2}$ line, but the lift velocity decays slower than $\left(a / x_{3}\right)^{2}$ at the values shown here. The rate of decay of the lift velocity decreases with decreasing capillary number. In addition, while the lift velocity at a quasi-steady state is proportional to the capillary number, the peak velocity of a capsule close to the wall is inversely proportional to the capillary number.

Figure 7 shows a spherical capsule with capillary number $C a=1.2$ and $\mathrm{RBCs}$ with a capillary number $C a=1.15$ with varying initial orientations. RBCs show a lift velocity profile that is nearly independent on the initial orientation and overlaps well with a capsule at a similar capillary number. Like the spherical capsule, the 


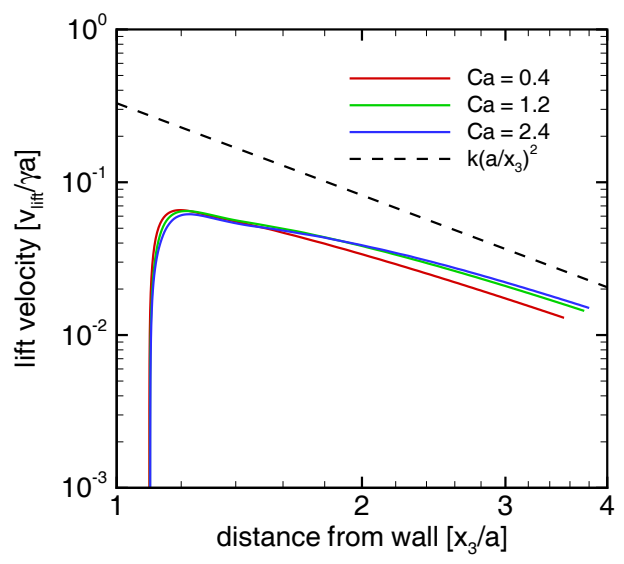

Figure 6: Lift velocity of spherical capsules.

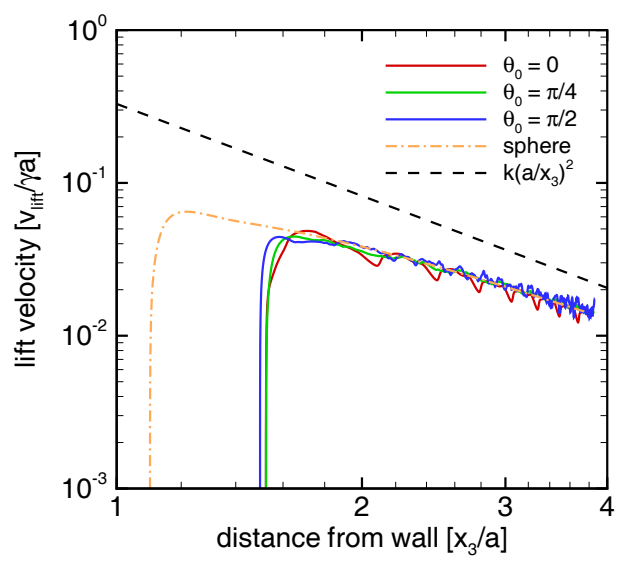

Figure 7: Lift velocity of a capsule and RBCs.

dependence of the lift velocity on the distance from the wall approaches $\left(a / x_{3}\right)^{2}$ at distances far from the wall, but as the RBC is moved closer to the wall, the decay of the lift velocity is slower than $\left(a / x_{3}\right)^{2}$.

\section{Discussion}

In the range of capillary numbers shown in figure 3 and figure 6 , a change in deformation results in only a modest change in lift velocity for a spherical capsule. A smaller change in the lift velocity occurs between $C a=1.2-2.4$ than that between $C a=0.4-1.2$. This can be explained by the observation that the change in deformation with respect to capillary number decreases as the capillary number increases. Since no lateral migration in low Reynolds number Couette flow is 
observed to occur for rigid spheres [12], the most significant variation in lift velocity is expected to occur at lower capillary numbers.

Both qualitative and quantitative changes in peak deformation between a spherical capsule and RBCs with different initial orientations are shown in figure 5. Despite these differences, the lift velocity profiles shown in figure 7 indicate that the transient deformation of a capsule, as well as its initial shape, had little effect on its rate of lateral migration. It seems that the steady state deformation of a spherical capsule determines the lift velocity of a capsule with the same capillary number, regardless of its initial shape.

While cells such as RBCs and WBCs occupy the same external flow conditions, $\mathrm{RBC}$ exhibit much higher deformability than WBCs. In other words, an RBC can be said to have a higher capillary number in the same flow as a WBC; thus, from this data, it is likely that RBCs experience a higher lift velocity than WBCs in the same external flow conditions. This is only one factor behind the mechanism of WBC margination; for example, the shear gradient present in a blood vessel is not examined here.

\section{Conclusion}

The deformation and lift velocity of spherical and biconcave capsules, models for WBCs and RBCs, were presented. While the peak and quasi-steady deformation profiles of capsules depend on initial shape, initial orientation, and capillary number, the lift velocity is not strongly affected by these transient effects. In addition, the lift velocity only shows strong dependence on capillary number at low $\mathrm{Ca}$. A difference in lift velocity between RBCs and WBCs may exist at sufficiently low capillary numbers, such as in microcirculation, and may be a factor in the initial margination of WBCs.

\section{References}

[1] Schmid-Schönbein, G.W., Usami, S., Skalak, R. \& Chien, S., The interaction of leukocytes and erythrocytes in capillary and postcapillary vessels. Microvascular Research, 19(1), pp. 45 - 70, 1980.

[2] Firrell, J. \& Lipowsky, H., Leukocyte margination and deformation in mesenteric venules of rat. American Journal of Physiology-Heart and Circulatory Physiology, 256(6), pp. H1667-H1674, 1989.

[3] Chaffey, C.E., Brenner, H. \& Mason, S.G., Particle motions in sheared suspensions. Rheologica Acta, 4, pp. 64-72, 1965.

[4] Chan, P.C.H. \& Leal, L.G., The motion of a deformable drop in a secondorder fluid. Journal of Fluid Mechanics, 92(01), pp. 131-170, 1979.

[5] Uijttewaal, W., Nijhof, E. \& Heethaar, R., Droplet migration, deformation, and orientation in the presence of a plane wall: A numerical study compared with analytical theories. Physics of Fluids A: Fluid Dynamics, 5, p. 819, 1993. 
[6] Uijttewaal, W.S.J. \& Nijhof, E.J., The motion of a droplet subjected to linear shear flow including the presence of a plane wall. Journal of Fluid Mechanics, 302, pp. 45-63, 1995.

[7] Blake, J.R., A note on the image system for a stokeslet in a no-slip boundary. Mathematical Proceedings of the Cambridge Philosophical Society, 70(02), pp. 303-310, 1971.

[8] Walter, J., Salsac, A.V., Barthès-Biesel, D. \& Le Tallec, P., Coupling of finite element and boundary integral methods for a capsule in a Stokes flow. International Journal for Numerical Methods in Engineering, 83(7), pp. 829$850,2010$.

[9] Skalak, R., Tozeren, A., Zarda, R. \& Chien, S., Strain energy function of red blood cell membranes. Biophysical Journal, 13(3), pp. 245-264, 1973.

[10] Evans, E. \& Fung, Y.C., Improved measurements of the erythrocyte geometry. Microvascular Research, 4(4), pp. 335-347, 1972.

[11] Ramanujan, S. \& Pozrikidis, C., Deformation of liquid capsules enclosed by elastic membranes in simple shear flow: large deformations and the effect of fluid viscosities. Journal of Fluid Mechanics, 361, pp. 117-143, 1998.

[12] Goldsmith, H. \& Mason, S., The flow of suspensions through tubes. I. Single spheres, rods, and discs. Journal of Colloid Science, 17(5), pp. 448-476, 1962. 